\title{
Photon energy dependence of graphitization threshold for diamond irradiated with an intense XUV FEL pulse
}

\author{
J. Gaudin, ${ }^{1,2, *}$ N. Medvedev, ${ }^{3}$ J. Chalupský, ${ }^{4}$ T. Burian, ${ }^{4}$ S. Dastjani-Farahani, ${ }^{2}$ V. Hájková, ${ }^{4}$ M. Harmand, ${ }^{5}$ \\ H. O. Jeschke, ${ }^{6}$ L. Juha, ${ }^{4}$ M. Jurek, ${ }^{7}$ D. Klinger, ${ }^{7}$ J. Krzywinski, ${ }^{8}$ R. A. Loch, ${ }^{9}$ S. Moeller,${ }^{8}$ M. Nagasono, ${ }^{10}$ \\ C. Ozkan, ${ }^{2}$ K. Saksl, ${ }^{11}$ H. Sinn, ${ }^{2}$ R. Sobierajski, ${ }^{7}$ P. Sovák, ${ }^{12}$ S. Toleikis,${ }^{5}$ K. Tiedtke, ${ }^{5}$ M. Toufarová, ${ }^{4,13}$ \\ T. Tschentscher, ${ }^{2}$ V. Vorlíček, ${ }^{4}$ L. Vyšín, ${ }^{4,13}$ H. Wabnitz, ${ }^{5}$ and B. Ziaja ${ }^{3,14}$ \\ ${ }^{1}$ Université de Bordeaux, CEA, CNRS, CELIA, UMR 5107, F-33400 Talence, France \\ ${ }^{2}$ European XFEL GmbH, Albert-Einstein-Ring 19, D-22761 Hamburg, Germany \\ ${ }^{3}$ Center for Free-Electron Laser Science, Deutsches Elektronen-Synchrotron DESY, Notkestrasse 85, D-22607 Hamburg, Germany \\ ${ }^{4}$ Institute of Physics, Academy of Sciences of the Czech Republic, Na Slovance 2, CZ-182 21 Prague 8, Czech Republic \\ ${ }^{5}$ HASYLAB/DESY, Notkestrasse 85, D-22607 Hamburg, Germany \\ ${ }^{6}$ Institut für Theoretische Physik, Goethe-Universität Frankfurt am Main, Max-von-Laue-Strasse 1, D-60438 Frankfurt, Germany \\ ${ }^{7}$ Institute of Physics Polish Academy of Sciences, Al. Lotników 32/46, PL-02-668 Warsaw, Poland \\ ${ }^{8}$ SLAC National Accelerator Laboratory, 2575 Sand Hill Road, Menlo Park, California 94025, USA \\ ${ }^{9}$ Dutch Institute for Fundamental Energy Research, P.O. Box 1207, NL-3430 BE Nieuwegein, The Netherlands \\ ${ }^{10}$ RIKEN/SPring-8 Kouto 1-1-1, Sayo, Hyogo 679-5148, Japan \\ ${ }^{11}$ Institute of Materials Research, Slovak Academy of Sciences, SK-04001 Kosice, Slovak Republic \\ ${ }^{12}$ Institute of Physics, P. J. Šafárik University, Park Angelinum, SK-04154 Kosice, Slovak Republic \\ ${ }^{13}$ Faculty of Nuclear Science and Physical Engineering, Czech Technical University, CR-11519 Prague 1, Czech Republic \\ ${ }^{14}$ Institute of Nuclear Physics, Polish Academy of Sciences, Radzikowskiego 152, PL-31-342 Krakow, Poland
}

(Received 8 March 2013; published 7 August 2013)

\begin{abstract}
We studied experimentally and theoretically the structural transition of diamond under an irradiation with an intense femtosecond extreme ultraviolet laser (XUV) pulse of 24-275 eV photon energy provided by free-electron lasers. Experimental results obtained show that the irradiated diamond undergoes a solid-to-solid phase transition to graphite, and not to an amorphous state. Our theoretical findings suggest that the nature of this transition is nonthermal, stimulated by a change of the interatomic potential triggered by the excitation of valence electrons. Ultrashort laser pulse duration enables to identify the subsequent steps of this process: electron excitation, band gap collapse, and the following atomic motion. A good agreement between the experimentally measured and theoretically calculated damage thresholds for the XUV range supports our conclusions.
\end{abstract}

DOI: 10.1103/PhysRevB.88.060101

PACS number(s): 81.05.ug, 81.30.-t, 61.80.Cb

It is often observed that a femtosecond irradiation of a material induces an atomic disorder therein: amorphization, or defect creation. Graphitization of diamond is a counterexample as it is an order-to-order (solid-to-solid) phase transition. It illustrates the fundamental interplay between the bonding, respectively $s p^{3}$ and $s p^{2}$ bonds for diamond and graphite, and the structure, respectively cubic and hexagonal. The advent of extreme ultraviolet (XUV) and x-ray free-electron lasers (XFELs), delivering femtosecond intense pulses in the soft to hard $\mathrm{x}$-ray domain allows investigating the structural transition of diamond within this unexplored regime and clarifying whether it leads to an ordered or disordered state. In our study we show the experimental results of the XUV irradiation of diamond followed by a dedicated theoretical analysis.

Irradiation by an optical femtosecond laser pulse triggers a specific process known as a nonthermal phase transition, which has been demonstrated for a class of materials. ${ }^{1}$ However, there are still active debates over the nature of the observed nonthermal transitions, e.g., see Ref. 2. For this type of transition, models predict that the excitation of a few percent of the valence band electrons leads to a drastic modification of the potential energy surface, triggering the displacement of the atoms. This process occurs on a much faster time scale (subpicosecond) than the transfer of the absorbed laser energy to the lattice via electron-phonon coupling. Such a nonthermal phase transition still needs to be observed in the $\mathrm{x}$-ray regime.
In the XUV and x-ray domain the excitation of electrons is only due to single photon absorption, and the absorption by free electrons does not occur. As a result, the first stage of the interaction, electron excitation and heating, which are driving nonthermal processes, is quite different compared to the optical regime. ${ }^{1}$ It is then questionable if a nonthermal phase transition can be triggered by an XFEL pulse. In the present Rapid Communication we identify the phase transition, which diamond undergoes under femtosecond XUV irradiation, as the graphitization. We show that the final state of the material after the laser pulse is ordered graphite, and the transition to this state is nonthermal.

In addition to this fundamental aspect, understanding the graphitization process is technologically relevant as diamond, with its different forms ranging from bulk, thin film, ${ }^{3}$ to nano-objects, ${ }^{4}$ is increasingly used for practical applications. As a result, graphitization, i.e., stability of diamond, upon annealing, ${ }^{5,6}$ high pressure, ${ }^{7,8}$ and irradiation by an ultrafast optical laser ${ }^{9,10}$ has been investigated, both experimentally and theoretically. The work presented in this Rapid Communication adds data for the phase-transition fluence threshold and an understanding of the response of diamond to intense XUV.

A first step to investigate this challenging issue was recently made in Ref. 11 with the development of a new theoretical hybrid model for the interaction of femtosecond XUV laser pulses with solids. The dynamics of the irradiated diamond 


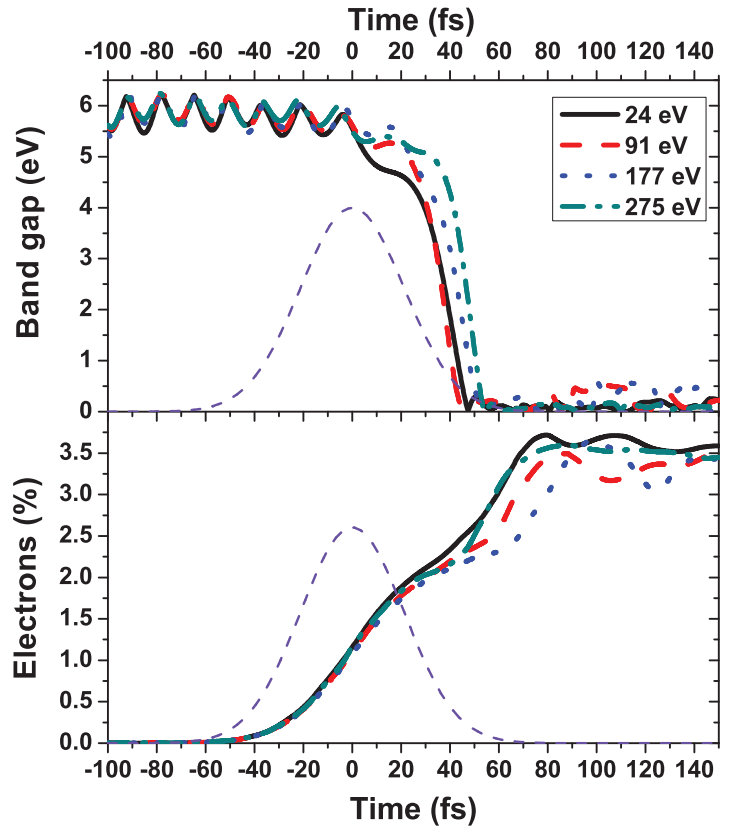

FIG. 1. (Color online) Calculated band gap (top) and conduction electron density relative to the total valence electron density (bottom) during the diamond-to-graphite phase transition after irradiation with the $50 \mathrm{fs}$ (FWHM) laser pulse with fluences yielding an average absorption of $0.7 \mathrm{eV} /$ atom for photon energies 24, 91, 177, and $275 \mathrm{eV}$. A Gaussian envelope of the laser pulse is schematically added.

is modeled by combining a nonequilibrium description of the ultrafast electronic excitation and relaxation with the atomic dynamics on a transient collective potential energy surface. For each transient atomic position, the new electronic band structure is also calculated. Such a combination allows us to follow any phase transition whether it is thermally or nonthermally activated. We use this model to describe the details of the XUV photon-induced phase transition of diamond for four different photon energies, including a prediction of the graphitization threshold.

For our analysis, we place the atoms initially on their equilibrium positions corresponding to the diamond structure, and assign to them velocities corresponding to room temperature. At room temperature, diamond has a band gap of $\sim 5.5 \mathrm{eV}$. This large band gap assures that there are nearly no conduction band electrons. After we let the atoms thermalize, i.e., establish a Maxwellian velocity distribution, we expose the simulation box to a laser pulse with a Gaussian intensity envelope of $50 \mathrm{fs}$ full width at half maximum (FWHM).

Figure 1 shows that the electron density within the conduction band increases, following photoabsorption and secondary impact ionization. This affects the atomic dynamics, and the electronic band structure. The band gap starts shrinking, as can also be observed in Fig. 1. Shrinkage of the band gap promotes more electrons from the valence to the conduction band, further enhancing the increase of conduction band electron density. Almost independently of the particular photon wavelength within the range from 24 to $275 \mathrm{eV}$, the band gap collapses within $\sim 40-50$ fs after the maximum of the laser pulse. This is accompanied by a further increase of the

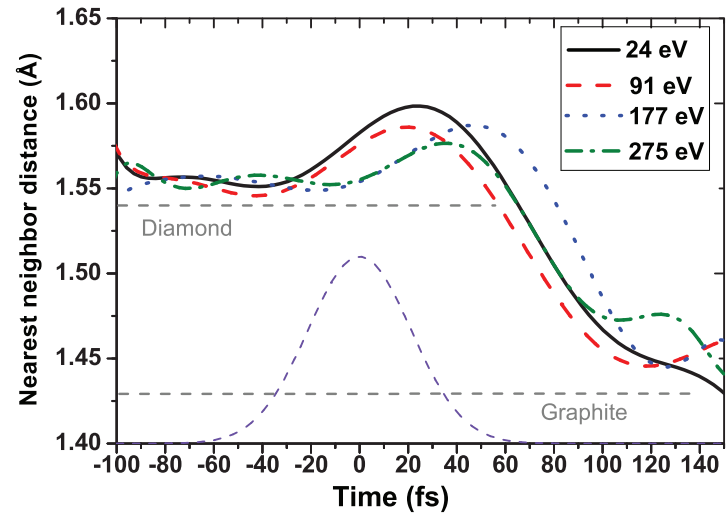

FIG. 2. (Color online) Calculated nearest neighbor distance between atoms during the diamond-to-graphite phase transition after irradiation with the $50 \mathrm{fs}$ (FWHM) laser pulse with fluences yielding an average absorption of $0.7 \mathrm{eV} /$ atom for photon energies of 24, 91, 177, and $275 \mathrm{eV}$. The horizontal lines, corresponding to the mean nearest neighbor distance for diamond and graphite, as well as the temporal FEL pulse profile, are shown as guides to the eye.

free-electron density. After this time, the electronic structure is already close to the one of graphite, rather than to the one of diamond.

The collapse of the band gap, and the corresponding increase of the conduction band electrons, changes the interatomic bonding. The atoms are affected by a new potential energy surface and move towards the new equilibrium positions, corresponding to the graphite phase.

This can be seen in Fig. 2, where the mean nearest neighbor distance is plotted as a function of time for different photon energies but at the same absorbed dose. One can see that shortly after the exposure, the diamond starts expanding. Later, following the band gap collapse at $\sim 40-50 \mathrm{fs}$ (compare Fig. 1), the relocation of atoms from diamond to graphite positions begins. It finishes within the next $40-50$ fs. Thus, within $\sim 80-100$ fs after XUV exposure, the irradiated diamond turns into graphite through nonthermal relaxation. The calculated atomic positions of the final state of the matter exactly correspond to the positions of graphite. ${ }^{12}$ The atomic positions obtained from the simulations clearly evidence a direct solid-to-solid phase transition, with no intermediate liquid state. Similar time scales for a nonthermal solid-to-liquid transition in graphite were recently reported in Ref. 13.

We have also calculated the dynamics of the irradiated diamond for different fluences, in order to identify the fluence threshold for graphitization. We find that the phase transition occurs when the conduction electron density overcomes a threshold value of $\sim 1.5 \%$ of the total number of valence electrons. When this occurs, the band gap collapses quickly and induces a further increase of the electron density, up to $\sim 3 \%$. This initiates the nonthermal phase transition. For all considered photon energies, i.e., 24, 91, 177, and $275 \mathrm{eV}$, the threshold has the same value of an absorbed energy of $0.7 \mathrm{eV} /$ atom. We conclude that this value is valid for the entire photon energy range between 24 and $275 \mathrm{eV}$.

In order to test this model, we have measured the graphitization thresholds experimentally. The experiments were performed at two different XFEL facilities: The experiments 


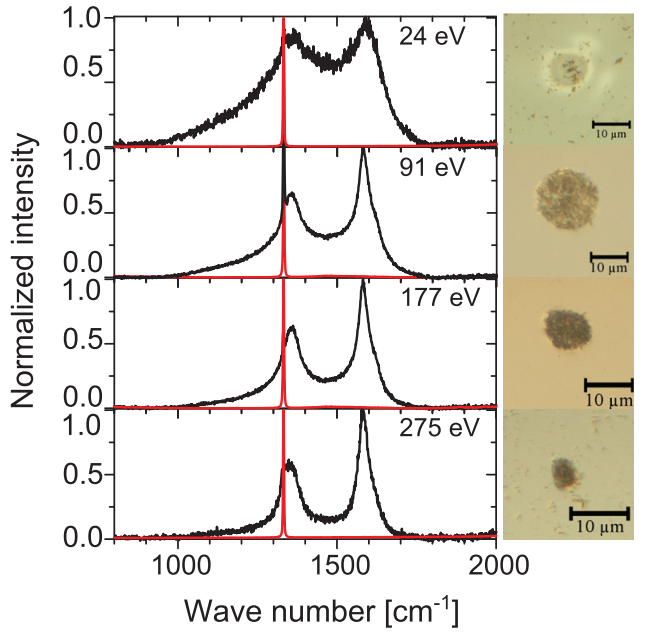

FIG. 3. (Color online) Normalized micro-Raman spectra for initial (red curves) and irradiated (black curves) diamond samples. Right: DIC pictures of samples irradiated by a single XFEL laser pulse corresponding to the micro-Raman measurements.

with $24 \mathrm{eV}$ photon energy were performed at the SPring-8 Compact SASE Source in Japan (SCSS) while the ones at 91, 177, and $275 \mathrm{eV}$ photon energies were performed at the Free Electron Laser in Hamburg (FLASH) in Germany. Both facilities deliver pulses of duration within the $30-80 \mathrm{fs}$ range. For all experiments, an experimental procedure was followed, which is described in detail in Ref. 14. The samples, consisting of chemical-vapor-deposited (CVD) diamonds, were placed in the focus of the focusing optics and exposed to a single XFEL pulse at normal incidence. The energy of each pulse was measured. The samples were analyzed ex situ using different techniques. First, each irradiated area was measured using a differential interference contrast (DIC) optical microscope (see the images in Fig. 3). This technique enables to measure any change of the optical refractive index and allows measuring the area of the x-ray induced modification. The measured areas are then plotted as a function of the impinging fluence. The experimental points are fitted down to 0 , i.e., no damage, which allows determining the phase-transition threshold fluence.

To gain a deeper insight in the effect of irradiation, microRaman spectroscopy measurements $(\lambda=514.5 \mathrm{~nm}$ laser, resolution $<4 \mathrm{~cm}^{-1}$ ) were performed on selected spots. Raman spectroscopy is a well-established technique, allowing measurements to be performed on a small surface ( $2 \mu \mathrm{m}$ spot diameter) and can unquestionably identify diamond and graphite phases. The red curve in Fig. 3 corresponds to the nonirradiated diamond sample and shows the typical peak of diamond at $1332 \mathrm{~cm}^{-1}$. The black curves correspond to the irradiated area at the center of the spot shown in the DIC pictures in Fig. 3. The Raman spectra feature two peaks located at $1575 \mathrm{~cm}^{-1}$ ( $G$ peak) and $1355 \mathrm{~cm}^{-1}$ ( $D$ peak). The $G$ peak is related to the degenerate transverse and longitudinal optical phonon mode of graphite, while the $D$ peak is related to defects in the graphite structure. ${ }^{15}$ The fact that both $G$ and $D$ peaks are clearly distinct indicates that the irradiation leads to graphite and not an amorphous structure. Moreover, the intensity ratio of $G$ and $D$ peaks is related to the size of the graphite crystallites. ${ }^{15}$ An ongoing quantitative analysis of this ratio as a function

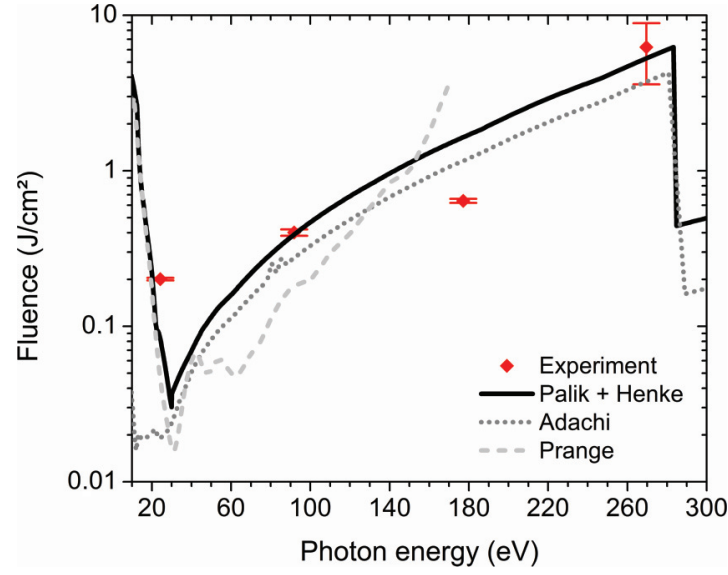

FIG. 4. (Color online) Fluence threshold for graphitization as a function of photon energy. Experimental points (red dots): Threshold determined by the model with different photoabsorption coefficients. Black line: Using coefficients from Ref. 11 for photon energies above $30 \mathrm{eV}$, and from Ref. 12 for the case of below $30 \mathrm{eV}$. The light gray dashed line uses the photoabsorption cross section from Ref. 21, and the dotted gray line is obtained with coefficients from Ref. 20.

of the impinging fluence shows that the size of the resulting graphite crystals is limited to a few nanometers. The high strain energy at the diamond/graphite surface is responsible for this size limitation, as in the case of thermally activated graphitization. ${ }^{6}$

The graphitization energy threshold is retrieved by extrapolating the DIC versus pulse energy data points down to zero. The full procedure is described in Ref. 14. The energy considered was the total energy arriving on the sample, i.e., taking into account the whole beamline transmission. In order to determine the fluence threshold, an estimate of the beam area is needed which is nontrivial as XFEL beam profiles are generally non-Gaussian. The effective area $A_{\text {eff }}$, which is equivalent to the area at the $1 / e$ level of a Gaussian beam, was determined by using a specific method described in detail in Ref. 16. Finally, the fluence threshold for graphitization is retrieved for each photon energy and is shown in Fig. 4. The larger error bar for the $275 \mathrm{eV}$ value is due to the uncertainty in the photon energy inducing a large uncertainty about the beamline transmission and impinging pulse energy, as explained in detail in Ref. 17.

The simulations predict a graphitization threshold of $0.7 \mathrm{eV} /$ atom, which is assumed to be valid for the energy range from 24 to $275 \mathrm{eV}$. In order to compare to the measured values, the dose has to be converted to fluence, which is achieved by taking into account the different photoabsorption cross sections of diamond provided in Refs. 18-21. The threshold predicted by theory is then represented in Fig. 4 by the continuous lines.

The measured thresholds are very close to the theoretical curves. This good agreement supports the validity of the model, and seems to indicate that the observed graphitization induced by XFEL pulses results from a nonthermal phase transition.

In conclusion, we have demonstrated, both experimentally and theoretically, that diamond undergoes a phase transition to graphite under XUV femtosecond irradiation. The simulations 
support the hypothesis of a nonthermal activation of the phase transition. In the framework of this model, we found that this transition proceeds as a multistep process. When the absorbed energy is high enough $(>0.7 \mathrm{eV} /$ atom $)$, the conduction electron density reaches $\sim 1.5 \%$. Such an electron density induces a band gap collapse. In turn, the electron density is further increased up to $\sim 3 \%$. This stimulates interatomic bond breaking, resulting in the phase transition. As the lattice temperature increases only after material modification (on the picosecond to nanosecond time scale), this also seems to support the nonthermal nature of the graphitization. We expect that our findings will then contribute to the ongoing discussion on the nature of nonthermal transitions.

The present study is limited to photon energies below the carbon $K$ edge $(\hbar \omega=285 \mathrm{eV})$. At photon energies higher than the $K$-edge energy the photoexcitation of core level electrons, and subsequent specific relaxation processes, ${ }^{22}$ such as Auger decay, would additionally influence the dynamics of the phase transition. Their specific contribution has to be investigated separately, both experimentally and theoretically.

We thank R. Santra for fruitful discussions and comments. K.S. and P.S. are indebted to the Slovak Grant Agency for Science for financial support (Grants No. 2/0128/13 and No. 1/0148/12). The Czech co-authors appreciate funding by Grants No. LG13029, No. CZ.1.07/2.3.00/30.0057, No. 13-28721S, No. P108/11/1312, No. P205/11/0571, No. P208/10/2302, and No. M100101221. R.S. was supported by the Polish National Science Center (Grant No. DEC2011/03/B/ST3/02453). H.O.J. is supported by the BeilsteinInstitut, Frankfurt/Main, Germany, within the research collaboration NanoBiC.
*Corresponding author: gaudin@ @elia.u-bordeaux1.fr

${ }^{1}$ S. K. Sundaran and E. Mazur, Nat. Mater. 1, 217 (2002).

${ }^{2}$ E. G. Gamaly and A. V. Rode, New J. Phys. 15, 013035 (2013).

${ }^{3}$ R. S. Balmer, J. R. Brandon, S. L. Clewes, H. K. Dhillon, J. M. Dodson, I. Friel, P. N. Inglis, T. D. Madgwick, M. L. Markham, T. P. Mollard, N. Perkins, G. A. Scarsbrook, D. J. Twitchen, A. J. Whitehead, J. J. Wilman, and S. M. Woolard, J. Phys.: Condens. Matter 21, 364221 (2009).

${ }^{4}$ A. Krueger, J. Mater. Chem. 21, 12571 (2011).

${ }^{5}$ M. Seal, Nature (London) 182, 1264 (1958).

${ }^{6}$ A. De Vita, G. Galli, A. Canning, and R. Car, Nature (London) 379, 523 (1996).

${ }^{7}$ Y. G. Gogotsi, A. Kailer, and K. G. Nickel, Nature (London) 401, 663 (1999).

${ }^{8}$ H. Chacham and L. Kleinman, Phys. Rev. Lett. 85, 4904 (2000).

${ }^{9}$ H. O. Jeschke, M. E. Garcia, and K. H. Bennemann, Phys. Rev. B 60, R3701 (1999).

${ }^{10}$ C. Z. Wang, K. M. Ho, M. D. Shirk, and P. A. Molian, Phys. Rev. Lett. 85, 4092 (2000).

${ }^{11}$ N. Medvedev, H. O. Jeschke, and B. Ziaja, New J. Phys. 15, 015016 (2013).

${ }^{12}$ See Supplemental Material at http://link.aps.org/supplemental/ 10.1103/PhysRevB.88.060101 for a snapshot of the atomic structure at different delays.
${ }^{13}$ S. P. Hau-Riege, A. Graf, T. Döppner, R. A. London, J. Krzywinski, C. Fortmann, S. H. Glenzer, M. Frank, K. Sokolowski-Tinten, M. Messerschmidt, C. Bostedt, S. Schorb, J. A. Bradley, A. Lutman, D. Rolles, A. Rudenko, and B. Rudek, Phys. Rev. Lett. 108, 217402 (2012).

${ }^{14}$ R. Sobierajski et al., J. Instrum. 8, P02012 (2013).

${ }^{15}$ S. Reich and C. Thomsen, Philos. Trans. R. Soc. London, Ser. A 362, 2271 (2004).

${ }^{16}$ J. Chalupský, J. Krzywinski, L. Juha, V. Hájková, J. Cihelka, T. Burian, L. Vyšín, J. Gaudin, A. Gleeson, M. Jurek, A. R. Khorsand, D. Klinger, H. Wabnitz, R. Sobierajski, M. Störmer, K. Tiedtke, and S. Toleikis, Opt. Express 18, 27836 (2010).

${ }^{17}$ J. Gaudin et al., Opt. Lett. 37, 3033 (2012).

${ }^{18}$ B. L. Henke, E. M. Gullikson, and J. C. Davis, At. Data Nucl. Data Tables 54, 181 (1993).

${ }^{19}$ E. D. Palik, Handbook of Optical Constants of Solids, Vol. I (Academic, Boston, 1998).

${ }^{20} \mathrm{~S}$. Adachi, The Handbook on Optical Constants of Semiconductors (World Scientific, Singapore, 2012).

${ }^{21}$ M. Prange, Ph.D. thesis, University of Washington, 2009.

${ }^{22}$ B. Ziaja, R. A. London, and J. Hajdu, J. Appl. Phys. 97, 064905 (2005). 